\title{
User Voice and Complaints as Drivers of Innovation in Public Services
}

\author{
Richard Simmons and Carol Brennan
}

Accepted for publication in Public Management Review by Taylor and Francis.

\begin{abstract}
User voice and complaints can serve as important inputs to innovation in public services. User knowledge can be harnessed to provide insights and ideas that prompt more effective service responses and add value to service delivery. However, the mechanisms for harnessing user voice and complaints are often not fully understood, and their potential is often underdeveloped. This paper elaborates a conceptual framework which maps the processes by which user voice and complaints might prompt effective public service innovation. Six practical real-world examples are then presented and analysed to illuminate discussion of some critical success factors for consumerknowledge-enabled innovation.
\end{abstract}

\section{Introduction: User Knowledge and Innovation in Public Services}

\section{Valuing Consumer Knowledge}

This paper considers user knowledge as a source of new ideas and creative solutions in today's public service environment. In the face of high expectations and relentless pressures to drive out costs, there is an ever-increasing need for smart, responsive public service provision, facilitated by cultures of innovation and improvement. The paper considers whether user voice and complaints can act as inputs for innovation by raising awareness, broadening and deepening discussion, and promoting strength in a diversity of ideas.

User voice can emerge from a range of sources, and a range of mechanisms have emerged for capturing and responding to it (Simmons et al, 2012). Users make choices about which channel for voice they consider appropriate for particular issues in a particular context: for example, through complaint and redress systems, contacting elected officials, participation in user groups/forums, or online mechanisms such as Fixmystreet or Patient Opinion (Simmons et al, 2012; Simmons \& Brennan, 2013). In some examples, user voice may also be solicited and structured through more interactive, co-productive initiatives, such as 'Experience-Based Co-design' (Bate \& Robert, 2007) or 'Experts by Experience' (Barnes, 2009). In this way, user voice can feed innovation in different ways for example, through the identification and expression of a 'disconfirmation' between expected and actual performance, or as an expression of consumer involvement and desire to 'co-produce' (Simmons, 2009).

Research shows people often have a relatively strong attachment to the public services they use, see them as important, and 'care about' them being done to a standard they find acceptable (Simmons et al, 2012). In this way, the legitimacy of user knowledge, whether expressed individually or collectively, lies both in users' informed evaluation of their own needs and their lived experience of 
public service use (Barnes, 2009; Centre for Public Scrutiny, 2011). Such conditions are important for the expression of voice (Hirschman, 1970; Dowding \& John, 2012).

In considering the role and potential of user voice and complaints, this paper builds on the emerging public service-dominant approach. For Osborne et al (2013), this approach raises concerns around the needs to: acknowledge public services as 'services' (and the centrality of the service user to their performance); incorporate citizen engagement and user involvement; and, therefore, enhance the capacity of public service organizations (PSOs) to understand the needs and expectations of citizens and service users in ways that add value. These concerns are followed through in identifying further needs for: creating a service culture and user-focused mindset; knowing users' expectations, requirements and value expectations; designing both the service and the experience of it; and measuring and assessing the service from the point of view of the user (Osborne et al, 2013; Osborne \& Strokosch, 2013). This in turn "requires both governing and responding to the service expectations of service users and training and motivating the service delivery workforce to interact positively with these users" (Osborne et al, 2013: 140). This is supported by effective communication practices/actions, whether intersubjective communication, or communicative action via other mediums. Respectful and clear communication plays a crucial role in establishing the more open and responsive organizational environments in which public service innovation can take hold.

Such perspectives begin to shift the focus from 'technical' to more 'relational' concerns. This reflects Hoggett's (2003) assessment that, 'the public sector is primarily a site for the enactment of particular kinds of social relations rather than the delivery of goods and services'. For Cloutier et al (2015), there is an important role here for relational work in the 'institutional work' done in PSOs. Institutional work has been defined as "the purposive action of individuals and organizations aimed at creating, maintaining and disrupting institutions" (Lawrence \& Suddaby, 2006: 215). Cloutier et al (2015: 21) found that institutional work comprises structural, conceptual, operational and relational components in PSOs, but that relational work serves to 'glue together' the other three forms and help PSOs navigate pluralism and contradiction. Such issues lead Hartley (2005: 27) to assert that innovation in public services 'is usually not a physical artefact at all, but a change in the relationships between service providers and their users'.

Such relationships provide scope for complementarities and creative solutions, but also for incompatibilities and conflicts of opinion. Hence, there is no guarantee that providers will agree over the appropriateness of voice and complaints in user-informed innovation (Bason, 2010; Simmons, 2011). Various well-known problems and pitfalls can arise. First, consideration must be given to who complains, how and how successfully. Some citizens do not even realise their entitlements, let alone have the agency to voice complaints about services they receive (e.g. Soss et al, 2011). This leads to the potential for complaint-led innovation to only make use of the knowledge of those most capable of pursuing their complaints. Second, and relatedly, the type and variety of mechanisms for gathering complaints matter. If these are either constrained, or made available but subsequently ignored, they can become tools of containment or exclusion (Simmons, 2009). In this sense, all three of Lukes' (1974) faces of power might apply: first, in terms of failing to uphold complaints, even when there are many of them; second, in terms of creating barriers to making complaints, so that few can be made; third, in terms of shaping a false consciousness in which people accept their role in the existing order of things and do not complain (Wood, 1995). In this sense, while one goal of better relationships might be to help public service organizations to manage better, the notion of 
nothing but good faith on their part cannot be assumed, and there is a need to maintain a variety of tools to help minimise and/or eliminate these issues (Simmons et al, 2012).

Indeed, Figure 1 shows how there is a range of potentially 'legitimate knowledges' in relation to public service issues. Each legitimate form of knowledge has its strengths and weaknesses. In this way, as Osborne \& Strokosch (2013) recognise, while it is clear that user-led transformative innovation can occur, it is not always straightforwardly-clear that all such innovations should occur; there is a need for wider debate. An important question here lies in the notion of 'boundary troubles' between different forms of knowledge. In short, who is it that decides when service innovation is called for - and are these the right people to do so?

\begin{tabular}{|l|l|}
\hline Form of knowledge & Critique \\
\hline Individual (e.g. Consumer) & 'Biased' \\
\hline Local (e.g. Local community) & 'Anecdotal' \\
\hline Specialised (e.g. Health professional) & 'Inaccessible' \\
\hline Strategic (e.g. Chief Executive) & 'Disconnected' \\
\hline Holistic (e.g. Academic professor) & 'Abstract' \\
\hline
\end{tabular}

Figure 1: Competing/Complementary Forms of Knowledge

Yet agreement and disagreement can each help prompt innovation (Cameron \& Freeman, 1991). A 'consumer knowledge management' approach (CKM; Gibbert et al, 2002) is offered here as tool for engaging with these issues and building 'integrative capacity' (Liao et al, 2009). The outset assumptions in CKM are that:

- Consumers have legitimate and distinctive knowledge that can contribute to service innovation, which may be 'managed' (acquired, absorbed, assimilated) alongside a range of other knowledges (Simmons \& Brennan, 2013)

- Such combinatory approaches may help contain any negative tendencies to overcustomization and/or inefficiency in public service innovations (Osborne et al, 2013)

Hence, CKM suggests moving beyond a plain one-way, administrative analysis of consumer knowledge, for example by analysing the data it holds on its consumers (including complaints statistics). It also contrasts with another commonly used term, consumer relationship management (CRM). While CRM may take consumer knowledge fully into account, it also encompasses a wellknown range of more negative approaches to the consumer-provider relationship - such as 'cooling the mark out' (Goffman, 1952) or 'therapy' and 'manipulation' (Arnstein, 1968). Instead, CKM 
explicitly recognises consumer knowledge to be as potentially significant to organizational decisionmaking as any other legitimate source. Acceptance of greater 'polyvocality' widens the repertoire of public services to deal with problems, and to promote innovation and improvement. Indeed, as Mulgan (2007: 4) observes:

'Users have always played a decisive role in social innovation - a role which is increasingly recognised in business too. In all cases, innovation usually involves some struggle against vested interests; the 'contagious courage' that persuades others to change; and the pragmatic persistence that turns promising ideas into real institutions'

Where PSOs are sufficiently open and responsive, this can help unlock the tacit or "sticky" knowledge that service users possess in order to improve existing or develop new services (Von Hippel, 1994, 2005; cf. Bason, 2010; Bessant \& Maher, 2009). This may involve a direct response to group voice, an individual communication, or the identification of trends in aggregate data (Simmons et al, 2012; Schibrowsky \& Lapidus, 1994). In this way, voice and complaints can help identify blind spots and dissonance in operational contexts, and foster innovation through the development of the new ideas and creative solutions (Bason, 2010).

Receptiveness to users' contributions acts as a catalyst for change. However, while public service users may be more likely to complain than exit if sufficiently convinced voice will be effective (Hirschman, 1986; Dowding \& John, 2012), research shows around one third feel they 'would not be listened to' (Simmons et al, 2012). Hence, as Osborne \& Strokosch (2013: S40) observe:

'It is not simply a case of empowering service users and expecting them to immediately begin transforming (public) services. Enhanced co-production requires a genuine partnership between public service professionals and service users that is predicated upon the use of knowledge to transform service delivery'.

Such openness and receptiveness from public service providers emphasises the overarching importance of greater competence, commitment and a more favourable organizational culture (Simmons \& Brennan, 2013). These issues underpin the analytical framework developed in this paper, which maps out how 'consumer-knowledge-enabled innovation' (CKEl; e.g. Belkahla \& Triki, 2011) may be promoted through better 'consumer knowledge management'.

The paper progresses as follows: first, the detailed analytical framework is presented. This considers (i) how PSOs go about valuing and managing knowledge through consumer knowledge management, and (ii) from this, how consumer-knowledge-enabled innovation may be captured from voice and complaints. Second, six practical real-world examples from the UK are described, and related to the framework. Finally, further discussion seeks to examine three key issues: the role and potential of user knowledge in public service innovation, PSOs' 'readiness' for CKEI, and the wider promotion of a public service-dominant approach.

\section{Analytical framework}

The analytical framework serves as a 'process map' by which user voice and complaints might prompt effective public service innovation. In this way, it stands as an intermediate step between a more general conceptual framework and a more detailed 'service blueprint' - the latter being a visualisation of service user and service staff actions/interactions that 'highlights the role(s) and 
relationship(s) of the service user within the service delivery system' through, for example, 'touchpoints', 'waitpoints' and 'failpoints' (Radnor et al, 2014).

The framework brings together the two related, but analytically-distinct, elements of 'Consumer Knowledge Management' (or how consumer knowledge is valued and managed; see Figure 2), and 'Consumer-Knowledge-Enabled Innovation' (or how innovation is captured from this process; see Figure 3). Combining these elements in the overall framework shows their inter-relationships in each of three notional 'systems' within public service organizations: the 'service delivery', 'innovation' and 'consumer knowledge management' systems (see Figure 4).

\section{Voice, Complaints and 'Consumer Knowledge Management' in Public Services}

The 'consumer knowledge management' element of the framework is concerned with an openlearning approach to valuing and managing consumer knowledge. Sometimes user voice and complaints are dismissed in PSOs, with people being labelled for example as 'the usual suspects' (e.g. Boswell et al, 2015) or 'frivolous' or 'vexatious complainants' (e.g. Hodges, 2015). While these stereotypes are now being challenged, and the reasons for users' persistence investigated more fully, there can remain a tendency in PSOs to 'fear rather than welcome value pluralism or conflict' (Hoggett, 2003: 3).

By contrast, a CKM approach regards voice and complaints as important inputs or prompts to innovation. Such inputs are elicited and encouraged through the above range of mechanisms to provide constant stimulation and insights. Leadbeater (2012) observes that these may:

- relate to either 'big stuff' (e.g. an innovation that saves $f 50000$ ten times per year) or 'small' stuff (e.g. an innovation that saves $f 5$ one hundred thousand times per year)

- be 'high volume' (i.e. those who 'shout the loudest') or 'low volume' (i.e. those who are 'hard to hear')

- involve 'many contributors' (collective voice/large aggregate numbers of complainants) or 'few contributors' (perhaps even a single individual).

The important thing is that the CKM process is able to find ways to capture the learning opportunities provided by these different inputs - even where, for example, they are 'low volume'. These may combine with 'other prompts' (especially those contributed by other legitimate knowledge bearers) in bringing about service innovation.

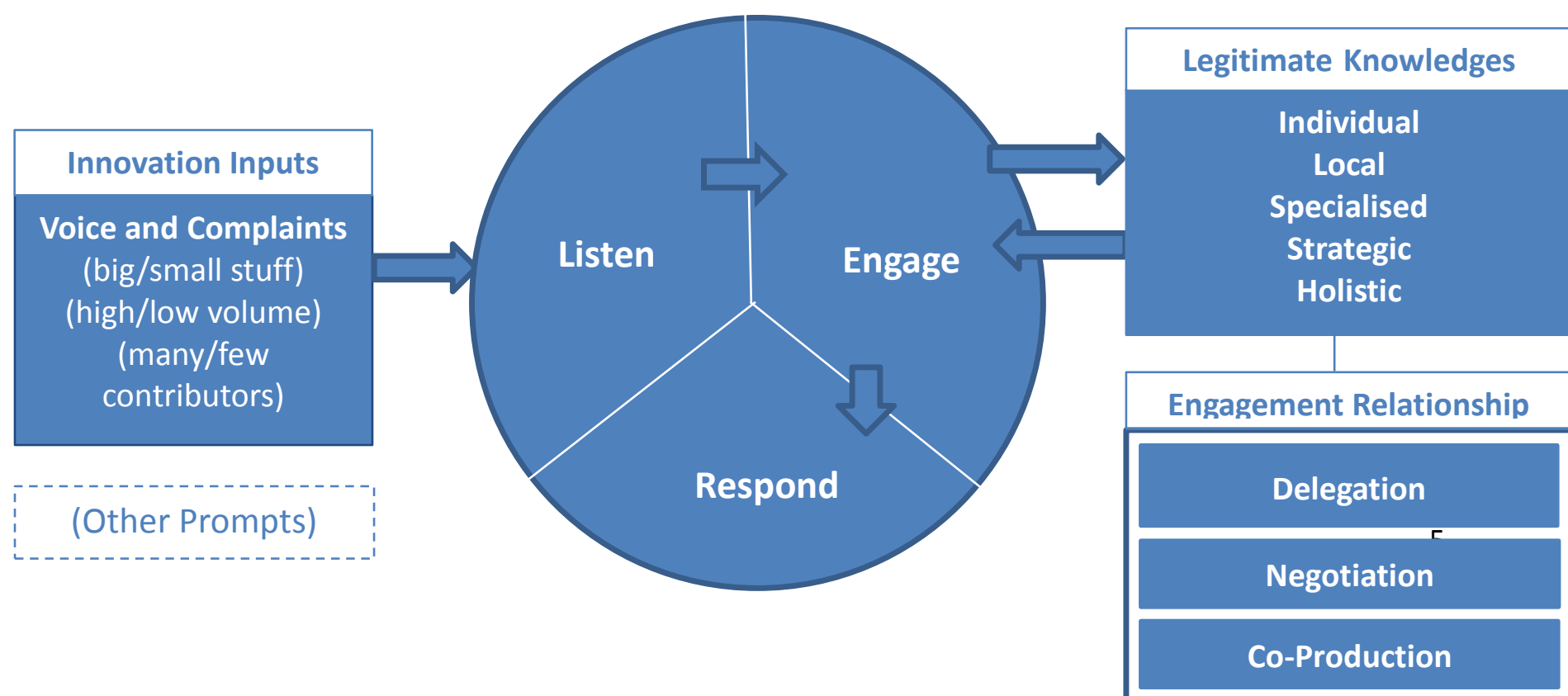


including complaints. However, as recognised by a former Departmental Permanent Secretary, "listening has not been one of the most precious public sector competencies, and too many managers are where they are because they are good at telling not listening" (Bichard, 2000). Good listening is a key facilitating factor for CKM - and poor listening a key constraining factor (Simmons, 2011).

'Engage' is the next stage of CKM. Here, the organization engages creatively with user inputs, where appropriate connecting with other legitimate knowledges in shaping a response. This recognises that no one source of knowledge has a monopoly on useful insights, and that each has its drawbacks and blind spots. The key here is including consumer knowledge in the mix. It is known from previous research that the 'engagement relationship' sought by public service consumers may vary (Simmons et al, 2012). Some may prefer to simply flag the issue then 'delegate' responsibility for finding a solution to public officials. Others may prefer to remain involved and 'negotiate' a solution with service providers (whereby providers come back to the user(s) with proposed actions and provide them with further opportunities to comment until agreement is reached). Still others may prefer to work alongside service providers in a more 'participative' or 'enhanced' co-productive approach (Osborne \& Strokosch, 2013) to help generate ideas and put those ideas into practice. More work remains to be done to consider the relative effectiveness of these different approaches on the extent to which voice and complaints help prompt innovation in public services.

The final step in the CKM model is 'respond'. It is at this point that that action begins to take shape. If user voice and complaints are assessed to be about service recovery, this may generate an appropriate response without resort to much that might be identified as 'innovation'. However, voice and complaints that go beyond simple service recovery represent a potential input into the innovation process. The next section of the paper will therefore address another key idea: 'Consumer Knowledge Enabled Innovation' (CKEI).

\section{Voice, Complaints and 'Consumer Knowledge-Enabled Innovation' in Public Services}

It was suggested earlier that the value of consumer knowledge in prompting innovation lies in raising awareness, broadening and deepening discussion, and acknowledging strength in a diversity of ideas. In this way, it has been asserted that user voice represents 'free consultancy' (e.g. Hobbs, 2014) or that 'a complaint is a gift' (Barlow \& Møller, 1996). This places an onus on the receivers of user knowledge to invest that endowment wisely. Once it has been combined with the insights from other legitimate knowledges, a central task is to translate these contributions into energy in support of three key innovation processes: 'invention', 'exploitation' and 'diffusion' (Rogers, 2003; Smith, 2010). These three processes imply productive and, to some extent, disruptive change (Bason, 2010).

In PSOs, such change may find its expression in service values, attributes (such as tangibles, systems, and practices) and/or consequences (Simmons et al, 2012). All are important. For example, Kelly et al (2002) emphasise that: 'values and value are closely linked. Inappropriate values may lead to the 
destruction of public value'. Meanwhile, in addition to tangible service attributes, Foss et al (2012) show that organizational systems are strategic resources that innovative organizations have begun to view proactively as necessary facilitators of success. Meanwhile, Hartley (2005) argues that the view of innovation as 'novelty in action' (Altschuler \& Zegans, 1997) and 'new ideas that work' (Mulgan \& Albury, 2003) focuses on innovation as new practice. Finally, the consequences of the service may also change, for example in the benefit they provide, for whom and with what impact.

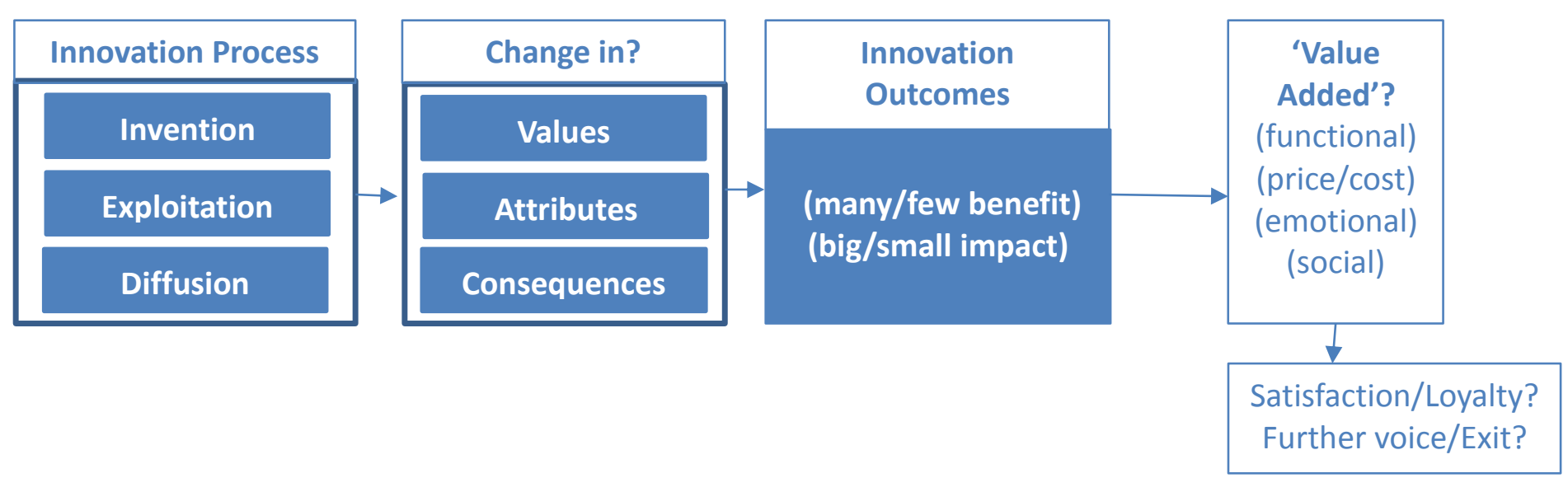

Figure 3: Capturing Innovation

In terms of outcomes, Figure 3 suggests that many people may benefit from service innovations, or just a few, and that this may have a relatively 'big' or 'small' impact. However, where a sufficient number of people benefit by a sufficient amount, a service innovation may be said to 'add value'. Value may be added in a number of important ways: 'financial' value (e.g. cost savings); 'functional' value (e.g. by calibrating public service activities to better fulfil the needs of users and citizens, and creating, sharing, transferring, adapting and embedding good practice; Hartley, 2005); 'social' value (e.g. wider non-financial impacts of programmes, organizations and interventions, including the wellbeing of individuals and communities, social capital and the environment; Wood \& Leighton, 2010) and/or 'emotional' value (e.g. through recognition, compassion, autonomy and care; Mulgan, 2007). Notably, the latter form of value is consistently raised as important by public service users in qualitative research into their experience (e.g. Simmons et al, 2012; 2013), but is rarely considered in standard assessments of value.

Finally, successful innovations that add value might be expected to develop user satisfaction (and, over time, even loyalty). However, where the innovation does not go far enough it may lead to further user voice and complaints, or even exit (Hirschman, 1970). This may prompt further rounds of attempted innovation. Hence, as shown in the bottom-left portion of Figure 4 below, the above elements of consumer-knowledge-enabled innovation may circulate in a loop until satisfaction is achieved.

Bringing Things Together: a 'three systems' framework 
Figure 4 brings the elements of CKM and CKEI together to show how they might be understood as part of the same whole. This heuristic framework shows three systems (each represented vertically): the 'service', 'innovation' and 'consumer knowledge management' systems.

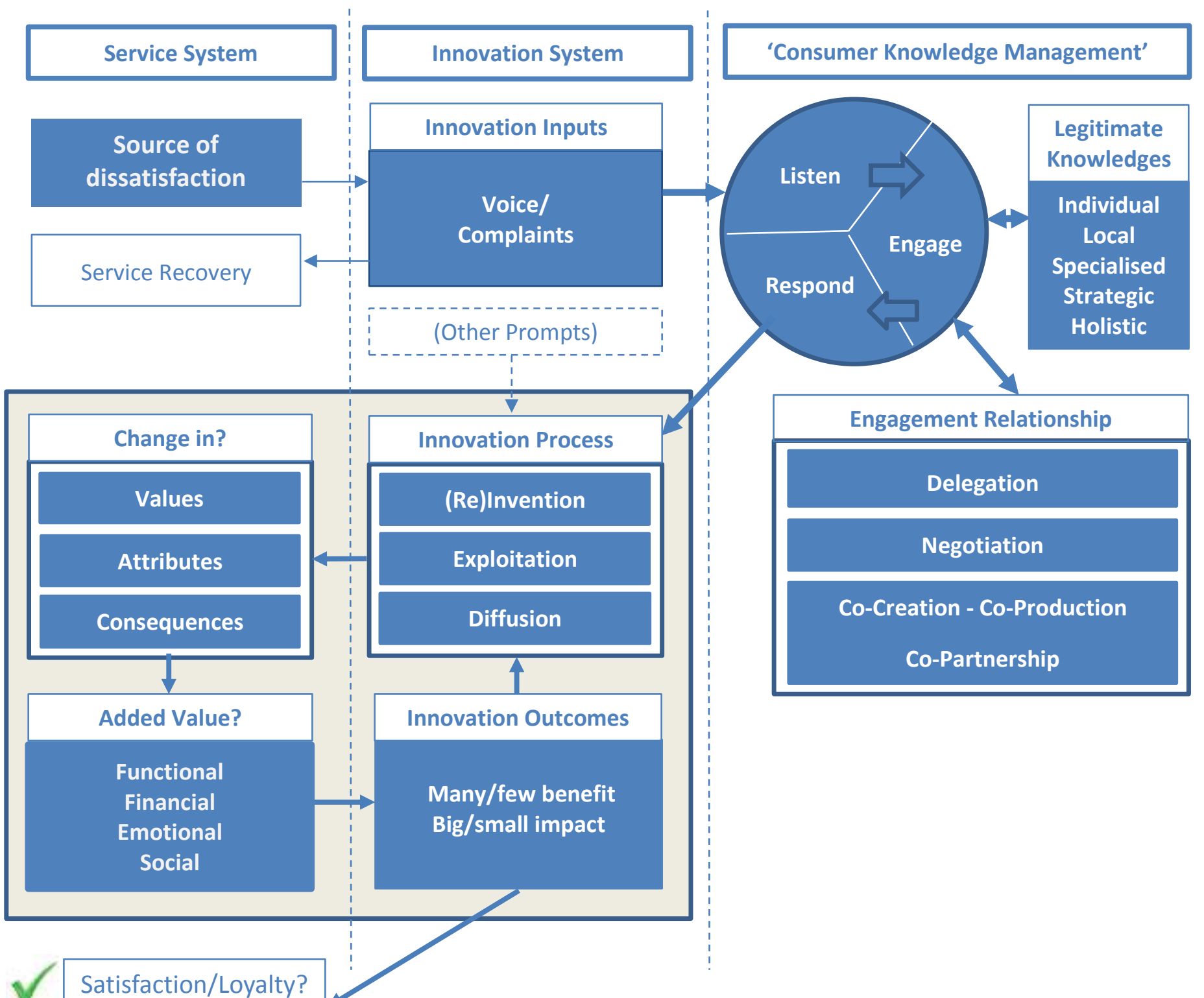

Figure 4: Integrated Analytical Framework

In terms of the elements of CKM and CKEI discussed above, some fit most clearly within the 'service system'. These include sources of dissatisfaction (including those that lead to simple service recovery), but also any changes in the values, attributes or consequences of the service that improve users' perceptions of value in ways that are necessary to rebuild their satisfaction. There are other elements that belong to the 'innovation system', whereby user voice and other inputs are used as resources for innovation processes that in turn produce innovative outcomes. The remainder fall within the realm of 'consumer knowledge management'. These include the cycle of listening, engaging different knowledges and responding appropriately. 
In sum, Figure 4 synthesises the key explanatory mechanisms through which user voice and complaints may help to drive public service innovation. Clearly, voice and complaints can serve as prompts for developing ideas. They also signify that providers do not have a monopoly on knowledge about public services. As understanding of these explanatory mechanisms continues to build, it is hoped this will illuminate some of the key ways in which complaints are translated into innovation processes, and with which outcomes.

\section{Disconnections between different elements}

Beyond this however, each link in Figure 4 provides insights into how opportunities for public service innovation may be lost. Starting at the top left, there is a risk that PSOs either fail to recognise the source of user dissatisfaction, or that it is incorrectly recognised and dismissed as a simple service recovery issue. Second, moving to the right, there is a risk that the legitimacy of user knowledge may be dismissed. Third, there is a risk that the communication is not properly listened to (perhaps because the receiver is self-preoccupied, closed-minded or 'too busy' to listen), so that its full implications are imperfectly understood. Fourth, there is a risk the PSO fails to engage the user further in clarifying and developing their communication. Fifth, there is a related risk that the engagement relationship is constructed on the wrong basis (perhaps due to a 'faulty default' assumption of delegation on the part of the service provider). Sixth, there is a risk of nonengagement of other legitimate knowledges, whether their input is directly sought or not. Seventh, there is a risk that even if these knowledges are engaged, they fail to make links with 'other prompts' that might add power and momentum to the process. Each of these risks may compromise the effectiveness of the eventual response.

To this may be added the generic risks associated with innovation processes in the bottom-left portion of Figure 4. Most of these have been widely discussed elsewhere. First, that creative ideas that lead to 'invention' may prove either unexploitable in particular contexts, or hard to diffuse (Rogers, 2003, Smith, 2010). This demands a level of risk tolerance in the pursuit of innovation that is not commonly found in PSOs (Albury, 2005). Second, that certain elements of organizations (e.g. values and culture) may be more impervious to change attempts than others (e.g. tangibles, systems and practices) (e.g. Harris \& Ogbonna, 2002). This may require particular investment in 'cultural innovation' (or a reprioritisation or rebalancing within organizational value systems that can help reframe the conceptual or emotional view of a situation, customize new strategies and promote new behaviours; Van Ess Coeling \& Simms, 1993) as part of the institutional work done by PSOs. Third, that any changes made may result in no - or insufficient - additions of value (or even the unintended consequence of lost value). It is widely recognised that such unintended consequences are all too common (Hood \& Peters, 2004).

At this point it should be noted that, associated with all the identified risks in Figure 4, there are countless real-life examples of failure of complaints to drive public service innovation, even where they may have had this potential. In this way, the framework does not only look to explain successes, but also failures to realise change (or why potential innovations might fail to emerge).

A further risk may be added; that the innovation process is abandoned too early, resulting in an opportunity cost. In this way, it may be prematurely accepted that an attempted innovation has failed, when further circulations of the loop in the bottom-left portion of Figure 4 may lead to its success. Hence, even where value is perceived to be added through an innovation, there remains the 
risk that this will be a sub-optimal response compared with that which might have been achieved with a little more effort.

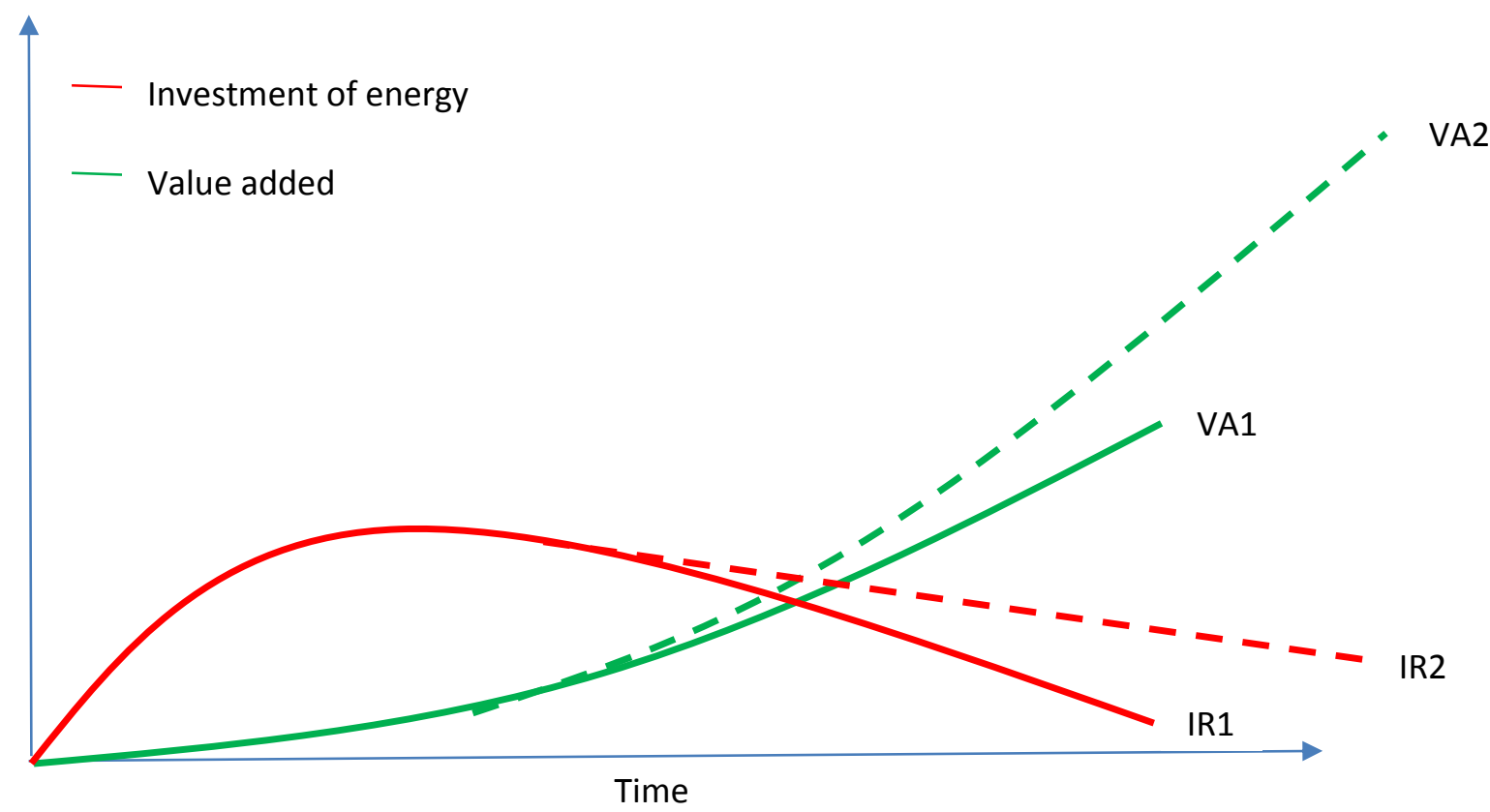

Figure 5: Return on investment of resources in CKEI

Figure 5 plots a hypothetical return over time on the investment of energy and knowledge in an innovation in terms of the value added. In this scenario a considerable investment of PSO resources is made early in the process (IR1) in understanding the problem, gathering information, engaging with stakeholders and so on, only for the response to be rushed in order for those resources to be redeployed back to their original purpose. As a result, the value added (VA1) is much less than that which might have been generated by seeing the job through (VA2), and continuing to invest a little more for a little longer (IR2). While this is a completely hypothetical scenario and many others are possible, the potential loss of net value it raises is very rarely considered in PSOs. Using the above framework to identify critical success factors, it is hoped this paper might encourage a more open, critical and reflective approach.

\section{CKEl: Six Practical Real-world Examples}

An extended process was followed to identify practical examples of CKEI in PSOs. This included a desk scan of online materials and crowdsourcing from organizations such as Patient Opinion and other public service contacts. The generally poor recording and/or celebration of success stories that this process uncovered meant that some promising examples were incomplete and needed to be excluded. Where more complete examples emerged from the above process, a number were selected for further attention. This included semi-structured interviews with key informants to probe for clarification and additional information. The six practical real-world examples included below 
have been compiled to exhibit a wide range of experiences and applications of CKEl. All examples are from the UK, and all relate to PSOs in which there are existing procedures for complaint handling. In each example, the framework has been employed to help identify critical success factors for user-led innovation in public services. This is clearly a small and unrepresentative sample, which is used to ground the framework in real-world dynamics. The six examples should therefore be seen as illustrations that provide a first test phase of the theoretical framework. In future, further research might be conducted using a more structured case study approach (e.g. Yin, 2013) to explore how the analytical framework might be applied (or even service blueprints developed) in relation to other real-world examples.

The following section commences with a brief description of each of the six examples. It then goes on to discuss and interpret these examples' success.

\section{The Six Practical Examples}

\section{A. 'Tell Us Once': Department for Work and Pensions (DWP)}

What was the complaint about? An elderly man was fatally run over outside the local post office. His frail and grieving widow attempted for months to cancel his pension, passport and so on. She repeatedly received inflexible responses from the authorities in a never-ending circle of bureaucracy. The final straw came when she was required to go in person to the same post office where her husband had died to cancel his pension.

What happened as a result? The eloquence and passion of her story prompted DWP to lead a partnership of central and local government departments to reduce the burden of bereavement. The result was a hugely innovative back-office fix called 'Tell-Us-Once' (TUO). The TUO service removes the need to separately notify different government services. Following registration of the death, a unique reference number is used to circulate details to all relevant government departments and services. The TUO Programme Manager, Matt Briggs, labels this 'a dynamic and inspiring model of how central and local government can work in true cooperation, smashing boundaries and prejudices with the hammer of a great idea'.

\section{B. Moffat Community Flood Resilience Group: Scottish Flood Forum (SFF)}

What was the complaint about? Lack of maintenance of drains is a major feature of flooding. Following repeated complaints, the community of Moffat, a small Scottish town, were angry with the public agencies who had failed to accept responsibility about flooding in the area for fear of litigation and insurance claims.

What happened as a result? In the absence of an adequate response from the statutory agencies, residents formed a 'Community Flood Resilience Group' to reduce their risk. They enlisted SFF, who adapted a US initiative to encourage members to each "adopt a drain". Members now check, monitor and notify the Council if drains need unblocking. They also use Twitter and Facebook to coordinate a timely collective response to flood risks and alert the authorities to respond. The Council is now fully engaged, in what has become a good example community empowerment and coproduction. Director of SFF, Paul Hendy, notes the importance of "confronting the issues and focusing the anger to develop creative solutions and rebuild confidence... Focusing on people's 
experiences changes the mindset - it's a totally new way of thinking". The approach has now been rolled out to other communities in Scotland and beyond.

C. Experience-Based Design (EBD): Luton and Dunstable Head and Neck Cancer Clinic (L\&D)

What was the complaint about? Patients complained about the insensitivity of being given a cancer diagnosis, then being rushed to further appointments about surgery, radiotherapy, a feeding tube which has to be cleaned, and so on - without time to digest their news or deal with the high emotion and distress.

What happened as a result? Short films of patients' stories were created and watched together by clinical staff and patients. These emotive films prompted deeper understanding of patients' care experiences and willingness to engage. Staff and patients then set about co-designing service change, finding new, innovative solutions to best meet patients' needs; e.g. rescheduling appointments to give patients more time, improving post-surgical training to be more responsive and producing a tracheostomy learning pack. L\&D CEO Stephen Ramsden points to more than 40 real service improvements directly from this work. Ward consultant, John Pickles, distinguishes the emotional insights from EBD as particularly important: "understanding how patients feel at certain points of their care leads us to the hard improvements, many of which we can do quickly and at little or no cost." EBD is now used extensively in the NHS and beyond.

\section{End-of-Life Care: SEQOL Swindon}

What was the complaint about? The daughter of a woman who had died complained about lack of communication with her and her family about her mother's end-of-life care. This resulted in their wishes not being taken into account.

What happened as a result? A long face-to-face meeting between the healthcare professionals and the family opened the provider's eyes to the insensitivity and inadequacy of the service system. Care plans were insufficiently robust; forms were jargon-filled and unfit for purpose, and there was poor co-ordination between primary and secondary care. A multi-agency end-of-life steering group was set up to look at the issues. This resulted in a new 'virtual ward' for end-of-life care, with a ward 'matron' to co-ordinate care plans, hospital admissions, outpatient appointments, and so on. However, when this solution was presented to the family, they were unhappy about not being able themselves to log their location, visiting times and so on. While giving families access to the NHS system proved 'challenging', all parties regathered their efforts to achieve what was agreed to be a vital extension to this innovation. According to the manager responsible, each meeting with the family was "really important in sparking people's ideas and turning our perspective around".

\section{E. Addiction Services: Patient Opinion (PO)}

What was the complaint about? An addict contacted PO about problems he experienced due to his Addictions Clinic being held on a Friday. Given his chaotic lifestyle, this meant that a missed clinic would leave him unable to access his medication before a 'mop-up' clinic on Monday. This had caused considerable personal distress and challenges. 
What happened as a result? The need for change was rejected initially, on the grounds of patients' personal responsibility to attend at the allotted time. However, the intervention of PO led gradually to a deeper engagement with the reality of these patients' lives. For Paul Hodgkin, Chair of PO, "the transparency of the web got people to act, rather than black-box the problem". It was suggested the clinic be moved to a Thursday, giving patients more time to access medication before the weekend. This saw drastic reductions in numbers at the 'mop up' clinic - previously stretched to capacity thereby also saving money. This is an excellent example of 'cultural' innovation and value change.

\section{F. Prison Super-complaint: National Consumer Council (NCC) and Prison Reform Trust (PRT)}

What was the complaint about? A prisoner complained to the Prisons and Probation Ombudsman that the cost of calls from prison payphones was 7 times more than the equivalent cost from a public payphone. This seemed unjustifiable at a time when telecommunications services were generally falling in price. This became an extremely long drawn out issue, which needed to overcome a culture of non-engagement with prisoners.

What happened as a result? The Ombudsman investigated and recommended changes, but the Prison Service rejected these recommendations. PRT and NCC then took on the issue. Eventually, this escalated into a 'super-complaint' to Ofcom (the UK telecommunications regulator). Values needed to shift (in another example of 'cultural innovation'), before service improvements could be achieved for this poorly-represented minority. All UK prison services have since agreed new telecoms contracts in line with market rates. Francesca Cooney from PRT says, "Prisoners can make more regular and longer calls to families and support systems outside the prison. This makes a huge difference, especially for those with mental health problems and suicidal concerns, providing a safer environment for prisoners. It is also important in reducing re-offending".

\section{Interpreting Change: mapping examples onto the framework}

Figure 6 attempts to map each example on to the framework in Figure 4. It also assesses which of the key risks each example had to overcome. This analysis shows that while each example of innovation was achieved in different ways, certain patterns also start to appear.

\begin{tabular}{|c|c|c|c|c|c|c|c|}
\hline \multicolumn{2}{|c|}{ Case Studies } & $\begin{array}{l}\text { Tell } \\
\text { Us } \\
\text { Once }\end{array}$ & $\begin{array}{l}\text { Community } \\
\text { Flood } \\
\text { Group }\end{array}$ & EBD & $\begin{array}{l}\text { End of } \\
\text { Life Care }\end{array}$ & $\begin{array}{l}\text { Addiction } \\
\text { Services }\end{array}$ & $\begin{array}{l}\text { Prison } \\
\text { Calls }\end{array}$ \\
\hline \multirow{5}{*}{$\begin{array}{l}\text { Knowledges } \\
\text { Included }\end{array}$} & Individual & $\mathbf{x}$ & $\mathbf{x}$ & $\mathbf{x}$ & $\mathbf{x}$ & $\mathbf{x}$ & $\mathbf{x}$ \\
\hline & Local & & $x$ & & & & \\
\hline & Specialised & $\mathbf{x}$ & $x$ & $\mathbf{x}$ & $\mathbf{x}$ & $x$ & $\mathbf{x}$ \\
\hline & Strategic & $\mathbf{x}$ & $\mathbf{x}$ & $x$ & $\mathbf{x}$ & $\mathbf{x}$ & $\mathbf{x}$ \\
\hline & Holistic & & & & & & \\
\hline & & & & & & & \\
\hline \multirow[b]{2}{*}{ Engagement } & Delegation & $\mathbf{x}$ & & & & & \\
\hline & Negotiation & & & & $\mathbf{x}$ & $\mathbf{x}$ & $\mathbf{x}$ \\
\hline
\end{tabular}




\begin{tabular}{|c|c|c|c|c|c|c|c|}
\hline Relationship & Co-Production & & $\mathbf{x}$ & $\mathbf{x}$ & & & \\
\hline \multirow{4}{*}{$\begin{array}{c}\text { What } \\
\text { Changes? }\end{array}$} & & & & & & & \\
\hline & Values & $\mathbf{x}$ & $\mathbf{x}$ & $\mathbf{x}$ & $\mathbf{x}$ & $\mathbf{x}$ & $\mathbf{x}$ \\
\hline & Attributes & $x$ & $x$ & $\mathbf{x}$ & $x$ & $x$ & $\mathbf{x}$ \\
\hline & Consequences & $\mathbf{x}$ & $\mathbf{x}$ & $\mathbf{x}$ & $\mathbf{x}$ & $\mathbf{x}$ & $\mathbf{x}$ \\
\hline \multirow{4}{*}{$\begin{array}{l}\text { Innovation } \\
\text { Outcomes }\end{array}$} & Many benefit & $\mathbf{x}$ & $\mathbf{x}$ & $\mathbf{x}$ & & & $\mathbf{x}$ \\
\hline & Few benefit & & & & $\mathbf{x}$ & $\mathbf{x}$ & \\
\hline & Big impact & $\mathbf{x}$ & $\mathbf{x}$ & $\mathbf{x}$ & $\mathbf{x}$ & $\mathbf{x}$ & $\mathbf{x}$ \\
\hline & Small impact & & & & & & \\
\hline \multirow{4}{*}{ Value Added } & Functional & $\mathbf{x}$ & $\mathbf{x}$ & $\mathbf{x}$ & $\mathbf{x}$ & $\mathbf{x}$ & $\mathbf{x}$ \\
\hline & Price/Cost & $x$ & $x$ & & & $x$ & $\mathbf{x}$ \\
\hline & Emotional & $x$ & $x$ & $\mathbf{x}$ & $\mathbf{x}$ & $x$ & $x$ \\
\hline & Social & $\mathbf{x}$ & $x$ & $x$ & $x$ & $x$ & $x$ \\
\hline \multicolumn{8}{|c|}{ Key Risks to Overcome } \\
\hline \multicolumn{2}{|c|}{ Failure to recognise problem } & $\mathbf{x}$ & $\mathbf{x}$ & $\mathbf{x}$ & $\mathbf{x}$ & $\mathbf{x}$ & $\mathbf{x}$ \\
\hline \multicolumn{2}{|c|}{$\begin{array}{c}\text { Legitimacy of user knowledge } \\
\text { dismissed }\end{array}$} & & & & & $\mathbf{x}$ & $\mathbf{x}$ \\
\hline \multicolumn{2}{|c|}{ User(s) not properly listened to } & $\mathbf{x}$ & $\mathbf{x}$ & $\mathbf{x}$ & $\mathbf{x}$ & $\mathbf{x}$ & $\mathbf{x}$ \\
\hline \multicolumn{2}{|c|}{ Failure to engage users in solution } & & & & $\mathbf{x}$ & $\mathbf{x}$ & $\mathbf{x}$ \\
\hline \multicolumn{2}{|c|}{$\begin{array}{l}\text { Engagement relationship } \\
\text { constructed inappropriately }\end{array}$} & & & & & $\mathbf{x}$ & $\mathbf{x}$ \\
\hline \multicolumn{2}{|c|}{$\begin{array}{c}\text { Failure to engage other legitimate } \\
\text { knowledges in solution }\end{array}$} & & & & & & $\mathbf{x}$ \\
\hline \multicolumn{2}{|c|}{ Failure to link to other prompts } & & & & & & $\mathbf{x}$ \\
\hline \multicolumn{8}{|c|}{$\begin{array}{l}\text { Ideas hard to exploit and/or } \\
\text { diffuse }\end{array}$} \\
\hline \multicolumn{2}{|c|}{ Imperviousness to change } & $\mathbf{x}$ & $\mathbf{x}$ & $\mathbf{x}$ & $\mathbf{x}$ & $\mathbf{x}$ & $\mathbf{x}$ \\
\hline \multicolumn{2}{|c|}{ Inability of change to add value } & & & & & & \\
\hline
\end{tabular}

Figure 6: Matrix of Framework Components and Associated Risks by Case Studies

First, individual (and sometimes local) user knowledge tends to combine with both specialist and strategic professional knowledges to find solutions. Each is an important link in the chain: user knowledges provide the spark for other actors; specialist knowledges help define whether a solution is actually possible or not; strategic knowledges provide the backing and support to see the innovation through. Any weak link in this chain may serve to threaten the innovation, or limit its impact.

Second, an appropriate engagement relationship was eventually found in each example. For 'Tell Us Once', the responsibility for envisaging and developing the back-office fix was necessarily delegated to others. In the 'End-of-Life' example, users negotiated the final solution with providers directly; in the 'Addiction Services' and 'Prison Calls' this negotiation was mediated by a trusted third party (PO and NCC/PRT respectively). Meanwhile, the 'EBD' and 'Flood Group' examples required more 'enhanced co-production' for their innovative outcomes to be achieved. 
Third, each example involves a change in values as well as service attributes (such as systems and practices) and consequences for users. In some examples the extent of value change is considerable; indeed, for the 'Addiction Services' and 'Prison Calls' examples, this was the main aspect in which innovation was required. Once this 'cultural innovation' had been achieved, the actual change in service attributes and outcomes was much more straightforward.

Fourth, each of the examples is identified as having a big impact, whether this is for 'the many' or 'the few'. In some examples ('Flood Group', 'EBD', 'Prison Calls'), impact for many has been achieved following the successful 'diffusion' of an initially more localised innovation.

This impact follows through in significant achievements of value in all examples, across all value categories. This is an important factor in the popularity of each of these innovations. In future, recognising value in all these different ways may help in at least two important respects: motivating action (due to seeing the potential benefits of innovation more broadly) and positively reinforcing innovative behaviours (due to broader acknowledgement of what has been achieved).

In terms of the potential risks, Figure 6 also shows how, in each example, there was a need to overcome at least an initial lack of provider recognition of the problem. This demonstrates the value of user knowledge in identifying issues from a unique perspective. In some examples, however ('Addiction Services', 'Prison Calls'), there continued to be a lack of provider recognition of a problem even when it had been clearly communicated to them by users. In all examples, there is also an identifiable failure to listen appropriately to users, at least initially. This was linked in all examples to PSOs' imperviousness to change. Overcoming this sometimes required the intervention of an enlightened professional or group of professionals ('Tell Us Once', 'EBD', 'End-of-Life'); in other examples the intervention of an enlightened third party ('Addiction Services', 'Prison Calls'); and in one example ('Flood Group'), direct action. However, the effort required in doing so was most obvious in two examples ('Addiction Services', 'Prison Calls'), where Figure 6 shows how many more of the risks to potential CKEI had to be overcome in eventually generating change. Beyond this, it should be noted that the value-addition from extended involvement in Figure 5 was most clearly visible in the 'End-of-Life' example, where the initially-proposed 'virtual ward' solution would have been suboptimal had it been implemented without further engagement with the family.

Finally, the above examples may also be considered in relation to how the boundaries between the three 'systems' in Figure 4 were managed. Once they had opened themselves up to the learning from user voice and the need for change had been accepted, some examples ('Tell Us Once', 'EBD') moved quickly and fluidly between the 'innovation' and 'CKM' systems to generate change (although much more co-productively in the example of EBD). While in the 'End-of-Life' example this progress was complicated by both the need for a multi-agency response crossing organizational boundaries and the need to engage IT knowledge in building the final solution, it is testament to the commitment of the SEQOL team and its partners that this process was seen through successfully. In the 'Flood Group' example, the local council appeared unwilling or unable (or both) to connect its systems with those of the group, until the group's action had become firmly established and the council could see a clearly identifiable 'win'. Even then, its initial engagement was predominantly through the 'service system' in separately supporting the group's activities with its own. Meanwhile, in the 'Addiction Services' and 'Prison Calls' examples, most of the work needed to be done in 'letting 
input in' (cf. the top half of Figure 4). Once this was accepted, actual service change was a relatively straightforward task.

The limitations of this study must be acknowledged; its findings are derived from a limited, if varied, range of experiences and applications of CKEI. While evaluation of the above examples suggests this paper's analytical framework provides new ways to understand the actions/interactions involved in successful CKEl, this remains to be tested further in future research. In the meantime, this paper suggests that user voice and complaints can act as inputs for innovation by raising awareness, broadening and deepening discussion, and promoting strength in a diversity of ideas. Work is now ongoing to develop the above framework into a simple checklist and/or app to guide the analysis of both formative and summative evaluations of the handling of user voice and complaints in different public service contexts. It is hoped that engagement of this more 'holistic knowledge' with practice will at least get stakeholders to consider different questions, and examine different perspectives, than otherwise might be the case.

\section{Conclusions}

This article draws together a series of inter-connected themes and evidence with regard to consumer-knowledge-enabled innovation in public services. In concluding this analysis, three seem worthy of note:

- The role and potential of user knowledge in catalysing change;

- The 'readiness' of PSOs for consumer-knowledge-enabled innovation and implications of this for 'institutional work'

- The contribution of these insights to the promotion of a public service-dominant approach

\section{Role and Potential of Consumer Knowledge}

This paper considers the role and potential of user voice in public services. It acknowledges that user voice can emerge from a range of sources, and that a range of mechanisms have emerged for capturing and responding to it (Simmons et al, 2012). This provides access to what nevertheless often remains an under-utilised source of knowledge. The practical real-world examples presented in the paper demonstrate the value of user knowledge in identifying issues from a unique perspective. The legitimacy of this knowledge, whether expressed individually or collectively, lies both in users' informed evaluation of their own needs and their lived experience of public service use (Barnes, 2009; Centre for Public Scrutiny, 2011). The examples presented in this paper also show how individual (and sometimes local) user knowledge can combine with both specialist and strategic professional knowledges to find innovative public service solutions. These innovations can have dramatic outcomes and add significant value, and many of them have been successfully 'diffused' to other contexts. However, such outcomes are not guaranteed. User knowledge has to connect with that of other legitimate stakeholders, many of whom hold their own perspectives on the issues at hand. The examples show how this sharing of territory provides scope for complementarities and creative solutions, but also how this requires incompatibilities and conflicts of opinion to be successfully overcome (cf. Simmons, 2011).

Readiness for CKEI and implications for 'Institutional Work' 
Some incompatibilities and conflicts of opinion result from competition between legitimate knowledges. Where, having fully considered the views of users, this competition results in the emergence of arguments that legitimately 'trump' users' concerns, it may be simply argued that the interests of 'procedural' and/or 'distributive' justice have been served.

However, such conditions are often unfulfilled. The six practical real-world examples show a common lack of provider recognition of the problem and failure to listen appropriately to users, at least initially. This was linked in all examples to an imperviousness to change, although most evidently in two examples ('Prison Calls' and 'Addiction Services'). Indeed, there was active resistance in these examples to acknowledging users' concerns, ostensibly based on strongly-held values about the rights and responsibilities of their respective client groups. In this way, the 'readiness' of some PSOs for CKEI appears greater than others in terms of their 'culture', 'competence' and 'commitment'. Taking these criteria in turn, first it would appear that in the most resistant examples, 'cultural innovation' and value change was required before further progress could be made. In other examples, once the initial barriers had been overcome, the cultural conditions were a little more complementary to the accommodation of user perspectives. Second, in at least one example ('Flood Group'), it would appear that the PSO also lacked the competence to respond to users' input with an effective innovation. This competence was gathered by users themselves in conjunction with a third party, before the innovation could be 'plugged back' into the overall service system for flood management. Finally, the commitment shown to pursue and maximise the potential of service innovation was exemplary in all examples: in terms of third parties in the 'Prison Calls' and 'Addiction Services' examples; service providers in the 'Tell Us Once' and 'End-of-Life' examples; service users in the 'Flood Group' example; and both providers and users in the 'EBD' example.

Moreover, in all practical real-world examples there was a need for the kind of 'institutional work' identified by Lawrence \& Suddaby (2006: 215). Cloutier et al's (2015) identification here of relational work as particularly important supports Hartley's (2005: 27) assertion that innovation in public services 'is usually not a physical artefact at all, but a change in the relationships between service providers and their users'.

\section{New perspectives on a public service-dominant approach}

In acknowledging such relational as well as technical concerns, the arguments and analysis in this paper help to elaborate new perspectives on the development of a public services-dominant approach. Given this approach's focus on the centrality of the service user to the performance of public services as 'services', and on incorporating citizen engagement and user involvement at all phases of a (public) service lifecycle (cf. Osborne et al, 2013), this paper addresses the capacity of public service organizations (PSOs) to understand the needs and expectations of citizens and service users in ways that add value. Moreover, in helping to specify the need for a service culture and userfocused mindset, and to know users' expectations, requirements and value expectations, the paper suggests a series of criteria that might be taken into consideration in (i) designing both the service and the experience of it (cf. Osborne et al, 2013), and (ii) measuring and assessing the service from the point of view of the user (cf. Osborne \& Strokosch, 2013). Moreover, this paper indicates a particular contribution from a broader understanding of 'value addition', using functional, financial, social and emotional criteria. Seeing the potential benefits of innovation in these various ways is 
more appropriate to the user experience, and advantageous in motivating and reinforcing positive, innovative behaviours. Finally, the paper responds directly to Osborne et al's (2013: 140) exhortations of "training and motivating the service delivery workforce to interact positively with these users". As stated above, the authors' engagement with stakeholders in developing tools such as checklists and apps is hoped to at least get them to consider different questions and examine different perspectives than otherwise might be the case. If so, it is hoped that more future consumer-knowledge-enabled innovations, such as those reported in this paper, might be expected in public service organizations.

\section{References}

Albury, D. (2005) 'Fostering Innovation in Public Services', Public Money and Management, 25 (1): 51-56

Altschuler, A. \& Zegans, M. (1997) 'Innovation and public management', In Innovation in American Government, Washington D.C.: Brookings Institution

Arnstein, S. (1968) 'A Ladder of Citizen Participation', Journal of the American Institute of Planners, 35: 216-244

Barlow, J. and Møller, C. (1996) A Complaint Is a Gift: Using Customer Feedback as a Strategic Tool. San Francisco: Berrett-Koehler Publishers

Barnes, M. (2009) 'Authoritative Consumers or Experts by Experience?', in Simmons, R., Powell, M. \& Greener, I. (eds.), The Differentiated Consumer in Public Services, Bristol: Policy Press

Bason, C. (2010) Leading Public Sector Innovation, Bristol: Policy Press

Bate, P. \& Robert, G. (2007) Bringing user experience to healthcare improvement: experience-based design, Oxford: Radcliffe

Belkahla, W. \& Triki, A. (2011) 'Customer knowledge enabled innovation capability: proposing a measurement scale', Journal of Knowledge Management, 15 (4): 648-674

Bessant, J. \& Maher, L. (2009) 'Developing Radical Service Innovations in Healthcare - the role of design methods', International Journal of Innovation Management, 13(4): 555-568

Bhalla, G. (2011) Collaboration and Co-Creation: New Platforms for Marketing and Innovation. New York: Springer

Bichard, M. (2000) 'The Modernization and Improvement of Government and Public Services: Creativity, Leadership and Change', Public Money \& Management, 20 (2): 41-46.

Boswell, J., Settle, C. \& Dugdale, A. (2015) 'Who Speaks, and in What Voice? The Challenge of Engaging 'The Public' in Health Policy Decision-Making', Public Management Review, 17(9)

Cameron, K. \& Freeman, S. (1991) 'Cultural Congruence, Strength and Type', Organizational Change and Development, 5:23-58

Centre for Public Scrutiny (2011) Aiming for the best, using lessons from complaints to improve public services, London: LGO

Cloutier, C., Denis, J-L., Langley, A. \& Lamothe, L. (2015) 'Agency at the Managerial Interface: Public Sector Reform as Institutional Work', J. Public Administration Research and Theory, online June 1: doi:10.1093/jopart/muv009 
Dowding, K. and John, P. (2012) Exits, Voices and Social Investment, Cambridge: Cambridge University Press.

Foss, N., Pedersen, T., Pyndt, J. \& Schultz, M. (2012) Innovating Organization and Management: New Sources of Competitive Advantage. Cambridge: Cambridge University Press

Gibbert, M., Leibold, M., \& Probst, G. (2002) 'Five Styles of Customer Knowledge Management, and How Smart Companies Use Them to Create Value', European Management Journal, 20: 459-469 Goffman, E. (1952) 'On Cooling the Mark Out: Some Aspects of Adaptation to Failure', Psychiatry: Journal of Interpersonal Relations, 15 (4):.451-463.

Harris, L. \& Ogbonna, E. (2002) 'The Unintended Consequences of Culture Interventions: A Study of Unexpected Outcomes', British Journal of Management, 13: 31-49

Hartley, J. (2005) 'Innovation in governance and public services: Past and present', Public Money and Management, 25, January: 27-34

Hirschman, A. (1970) Exit, Voice, and Loyalty: responses to decline in firms, organizations, and states, Cambridge, Mass.: Harvard University Press

Hirschman, A. (1986) 'Exit and Voice. An Expanding Sphere of Influence', in: Hirschman, A. (ed.) Rival Views of Market Society, New York: Viking

Hobbs, G. (2014) 'Delivering Continuous Improvement in Government', paper to Open Forum Workshop: Continuous Improvement: Inspiring Better Public Services, Manchester, 28th May Hodges, C (2015) 'Consumer Ombudsmen: better regulation and dispute resolution', ERA Forum: Journal of the Academy of European Law, 15(4): 593-608

Hoggett, P. (2003) 'Why Public is not the same as Private', paper to The International Society for the Psychoanalytic Study of Organizations Symposium, Power and Politics, Boston, June 19-21.

Hood, C. \& Peters, G. (2004) 'The Middle Aging of New Public Management: Into the Age of Paradox?' Journal of Public Administration Research and Theory, 14 (3): 267-282

Kelly, G., Mulgan, G., Muers, S. (2002) Creating Public Value: An analytical framework for public service reform, London: Strategy Unit

Lawrence, T. \& Suddaby, R. (2006) 'Institutions and Institutional Work', in S. Clegg et al (eds.)

Handbook of Organizational Studies, London: Sage

Leadbeater, C. (2012) Learning from the Extremes: strategies for radical social innovation, Internal presentation, NESTA

Liao, J., Kickul, J. \& Ma, H. (2009) 'Organizational dynamic capability and innovation: an empirical examination of internet firms', Journal of Smart Business Management, 47 (3): 263-86.

Lukes, S. (1974) Power: a radical view, London: Macmillan

Mulgan, G. (2007) Ready or not? Taking innovation in the public sector seriously, London: NESTA.

Mulgan, G. and Albury, D. (2003) Innovation in the Public Sector, London: Cabinet Office.

Osborne, S., Radnor, Z. \& Nasi, G. (2013) 'A New Theory for Public Service Management? Towards a

(public) service-dominant approach', American Review of Public Administration, 43: 135-158

Osborne, S. \& Strokosch, K. (2013) 'It takes two to tango? Understanding the co-production of public services by integrating the services management and public administration perspectives', British Journal of Management, 24: S31-S47 
Radnor, Z., Osborne, S., Kinder, T. \& Mutton, J. (2014) 'Operationalizing Co-Production in Public Service Delivery: The contribution of service blueprinting', Public Management Review, 16 (3), 402423

Rogers, E. M. (2003) Diffusion of Innovations (5th ed.), New York: Free Press

Schibrowsky, J. \& Lapidus, R. (1994) 'Gaining a Competitive Advantage by Analyzing Aggregate Complaints', Journal of Consumer Marketing, 11 (1): 15-26

Simmons, R. (2009) 'The Differentiated Consumer in Public Services', in Simmons, R., Powell, M. \& Greener, I. (eds.), The Differentiated Consumer in Public Services, Bristol: Policy Press

Simmons, R. (2011) 'Leadership and Listening in Public Services', Social Policy and Administration, 45(5):539-568

Simmons, R. (2013) Complaints and Innovation in Public Services, paper to Policy and Politics International Conference Transforming Policy \& Politics: The Future of the State in the 21st Century, Bristol, 17-18 September

Simmons, R., Birchall, J. \& Prout, A. (2007) 'Hearing Voices? User Involvement and Public Service Cultures', Consumer Policy Review, 17 (5): 234-240

Simmons, R., Birchall J. \& Prout, A. (2012) 'User Involvement in Public Services: 'choice about voice", Public Policy and Administration, 27(1): 3-29

Simmons, R. \& Brennan, C. (2013) Grumbles, gripes and grievances: the role of complaints in transforming public services, London: Nesta.

Simmons, R., Brennan, C., Gill, C. \& Hirst, C. (2013) Outcomes of Complaints, Perth: Care Inspectorate Smith, D. (2010) Exploring Innovation (2nd ed.), Maidenhead: McGraw-Hill Education Soss, J., Fording, R \& Schram, S. (2011) Disciplining the Poor, Chicago: Univ. Chicago Press Van Ess Coeling, H. \& Simms, L. (1993) 'Facilitating Innovation through Cultural Assessment' Journal of Nursing Administration, 23(4): 46-53

Von Hippel, E. (1994) 'Sticky information and the locus of problem solving: implications for innovation', Management Science, 40: 429-439.

Von Hippel, E. (2005) Democratizing Innovation, Cambridge, MA: MIT Press.

Wood, C. Leighton, D. (2010) Measuring Social Value: the gap between policy and practice, London: DEMOS

Wood, D. (1996) 'Acting on complaints about mental health services: Implications of power imbalances', Journal of Management in Medicine, 10 (3), 31-38

Yin, R. (2013) Case Study Research: design and methods (5 $5^{\text {th }}$ ed.), London: Sage 